\title{
Effect of high-dose pancreatic enzyme replacement on prevention of postpancreatectomy hepatic steatosis after pancreatoduodenectomy: A prospective cohort study
}

\author{
Moonhwan KIM', Yoo-Seok YOON*1, Jin-Young JANG ${ }^{2}$, Wooil KWON², Hongbeom KIM², Dong-Sup YOON ${ }^{3}$, \\ Joon Seong PARK ${ }^{3}$, Seung Eun LEE ${ }^{4}$, Jun Suh LEE', Ho-Seong HAN' \\ 'Department of Surgery, Seoul National University Bundang Hospital, Seoul National University College of Medicine, Seoul, Korea \\ ${ }^{2}$ Department of Surgery, Cancer Research Institute, Seoul National University College of Medicine, Seoul, Korea \\ ${ }^{3}$ Department of Surgery, Gangnam Severance Hospital, Seoul, Korea \\ ${ }^{4}$ Department of Surgery, Chung-Ang University Hospital, Seoul, Korea
}

Introduction: Postpancreatectomy hepatic steatosis (PHS) is a long-term complication of pancreaticoduodenectomy (PD), which has been not well noticed. The aim of this study was to evaluate the effect of high-dose pancreatic enzyme replacement therapy (high-dose PERT) on prevention of PHS after PD by analyzing prospectively collected data.

Methods: Among patients who were assigned randomly to receive high-dose PERT (40,000 IU of Norzyme) for 3 months or placebo in the previous prospective study (Clin Gastroenterol Hepatol. 2020;18:926-934), 94 patients who had unenhanced computer tomography $(\mathrm{CT})$ scans at preoperative and postoperative 1 year were enrolled in this study. PHS was defined when mean CT values of the liver were $<40$ Housnfield unit (HU) or $10 \mathrm{HU}$ lower than values of the spleen. The incidence and risk factors of newly developed PHS at postoperative 1 year were analyzed.

Results: There were 42 patients in the high-dose PERT group and 52 patients in the placebo group. The incidence of PHS was significantly lower in the high-dose PERT group (4/42) compared with the placebo group (12/52) $(p<0.001)$. Multivariate analysis identified placebo intake (odds ratio [OR] 1.640, $p=0.020$ ), preoperative body mass index (BMI) $>24(\mathrm{OR} 9.065, p=0.032)$, body weight loss $>5 \%$ in postoperative 3 months (OR 2.087, $p=0.024$ ) as independent risk factors. Subgroup analysis showed that the lower incidence of PHS after high-dose PERT compared with the placebo control were consistent in high risk groups: preoperative BMI $>24$ (high-dose PERT vs. placebo, $3 / 14$ vs.13/22, $p=0.019)$ and body weight loss $>5 \%(4 / 12$ vs. $30 / 46, p=0.013)$.

Conclusions: The results revealed that high-dose PERT significantly reduced the development of PHS after PD. 